Received: 2015.10.13

Accepted: 2015.11.12

Published: 2016.02.19

\title{
Primary Breast Lymphoma in a Woman: A Case Report and Review of the Literature
}

Authors' Contribution

Study Design A

Data Collection B

Statistical Analysis C

Data Interpretation D

Manuscript Preparation E

Literature Search F

Funds Collection $G$
ABEF 1 Gaby Jabbour

ABEF 1 Gamela El-Mabrok

ABEF 1 Hassan Al-Thani

BDEF 2,3 Ayman El-Menyar

BDE 4 Ibrahim Al Hijji

BDE 5 Sarbar Napaki
1 Department of Surgery, General Surgery, Hamad General Hospital, Doha, Qatar 2 Department of Surgery, Clinical Research, Hamad General Hospital, Doha, Qatar 3 Internal Medicine, Weill Cornell Medical College, Doha, Qatar 4 Department of Hematology, Hamad General Hospital, Doha, Qatar 5 Department of Laboratory Medicine and Pathology, Hamad General Hospital, Doha, Qatar
Corresponding Author: Conflict of interest:

\begin{tabular}{|c|c|}
\hline Patient: & Female, 43 \\
\hline Final Diagnosis: & Primary breast lymphoma \\
\hline Symptoms: & - \\
\hline Medication: & - \\
\hline Clinical Procedure: & CT scan • PET • chemotherapy • radiotherapy \\
\hline Specialty: & Oncology \\
\hline Objective: & Rare disease \\
\hline Background: & $\begin{array}{l}\text { Primary breast lymphoma (PBL) is an unusual clinical entity accounting for } 0.4-0.5 \% \text { of all breast neoplasms. } \\
\text { The usual presentation includes a painless palpable mass similar to that of breast carcinoma. Diffuse large B-cell } \\
\text { lymphoma (DLBCL) is the most common identifiable type of PBL based on the histopathological examination. }\end{array}$ \\
\hline Case Report: & $\begin{array}{l}\text { We report an unusual case of a } 43 \text {-year-old Indonesian woman who presented with a } 7 \text {-month history of a } \\
\text { painless mass in the left breast. A core needle biopsy revealed diffuse infiltration of large atypical lymphoid } \\
\text { cells. The immuno-histochemical biomarkers confirmed the diagnosis of a DLBCL. A bone scan showed no ev- } \\
\text { idence of bone metastasis. It was treated non-surgically, based on the decision of the breast multidisciplinary } \\
\text { team (MDT). The patient was treated with } 4 \text { cycles of combination chemotherapy with R-CODOX/IVAC. A fol- } \\
\text { low-up PET scan revealed non-significant mild F-18 fluorodeoxyglucose (FDG) uptake at the periphery of the } \\
\text { residual left breast mass, indicating a radiologically favorable response. }\end{array}$ \\
\hline Conclusions: & $\begin{array}{l}\text { Early and accurate diagnosis of PBL is crucial for selecting the appropriate MDT treatment strategies to avert } \\
\text { potentially harmful surgical interventions. }\end{array}$ \\
\hline
\end{tabular}

MeSH Keywords: Breast Neoplasms • Fluorodeoxyglucose F18 • Lymphoma, B-Cell

Full-text PDF: http://www.amjcaserep.com/abstract/index/idArt/896264

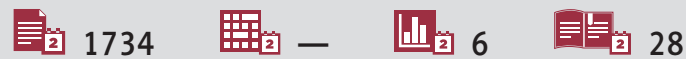




\section{Background}

Primary breast lymphoma (PBL) is an unusual tumor accounting for $0.4-0.5 \%$ of all breast carcinomas [1]. It is a rare neoplasm because lymphoid tissue is absent in the breast region. The neoplasm can develop in both sexes, but it is more frequent among females. Breast lymphoma has been classified into primary and secondary types. $\mathrm{PBL}$ is defined as the presence of both mammary tissue and lymphoid infiltrate in close association with no evidence of widespread lymphoma or preceding extra-mammary lymphoma [2,3]. It has been reported that high-grade lymphomas commonly manifest as diffuse breast enlargement, whereas low and intermediategrade tumors often have nodular patterns $[4,5]$. PBL is usually non-Hodgkin's B-cell type, which accounts for around half of breast lymphomas, and the diffuse large B-cell lymphoma $(\mathrm{DLBCL})$ is the most frequent subtype $[6,7]$.

The usual presentation of PBL is painless palpable mass similar to that of breast carcinoma. Patients with PBL infrequently present with signs of skin edema, retraction, erythema, and nipple involvement [8]. The confirmed diagnosis of PBL is mainly based on findings of the initial biopsy or postoperative pathology results [8].

The treatment options for PBL vary from surgical intervention to chemotherapy and/or radiotherapy [9]. Herein, we report a rare case of primary non-Hodgkin lymphoma of the breast, with B-cell phenotype, diagnosed by mammography and computed tomography and confirmed by histopathological examination. It was successfully treated non-surgically according to the breast multidisciplinary team (MDT).

\section{Case Report}

A 43-year-old Indonesian woman, previously healthy, presented with a left breast lump that became noticeable 7 months before. The mass had rapidly increased in size. She had no pain or retraction, no skin changes, and no nipple discharge. Her family history was negative for breast cancer. She was married, menstruating, and had 2 children. Physical examination revealed a hard, non-tender mass involving the whole left breast with a $2 \times 1.5 \mathrm{~cm}$ palpable left axillary node. Radiological imaging (mammogram and ultrasound) and histopathological investigation (fine-needle aspiration (FNA) and core biopsy) were performed. The mass was staged with computerized tomography (CT) scan chest/abdomen, and bone scanning. The ultrasonography findings revealed a large, ill-defined, dense mass with internal increased vascularity in color Doppler imaging and multiple enlarged, hypoechoic, axillary lymph nodes (Figure 1). These findings were highly suggestive of breast carcinoma with metastatic axillary lymph nodes. Mammography findings were also suggestive of breast cancer with metastatic axillary lymph nodes; BI-RADS 5 (Figure 2). A bone scan showed no evidence of bone metastasis. The positron emission tomography (PET) scan demonstrated intense hypermetabolism in the left breast, indicating diffuse large B-cell lymphoma (DLBCL). Fine-needle aspiration of the left axillary lymph node showed atypical lymphoid cells suggestive of malignancy. The histopathology findings confirmed the diagnosis with stage IV BEx DLBCL; active B-cell type. Hematoxylin and eosin staining showed large atypical lymphoid cells in sheets (Figure 3). Figures 4 and 5 demonstrate the immunohistochemistry profile. The lymphoid cells showed diffuse cytoplasmic staining for CD20, CD79, and BCL2. There was nuclear staining for BCL6

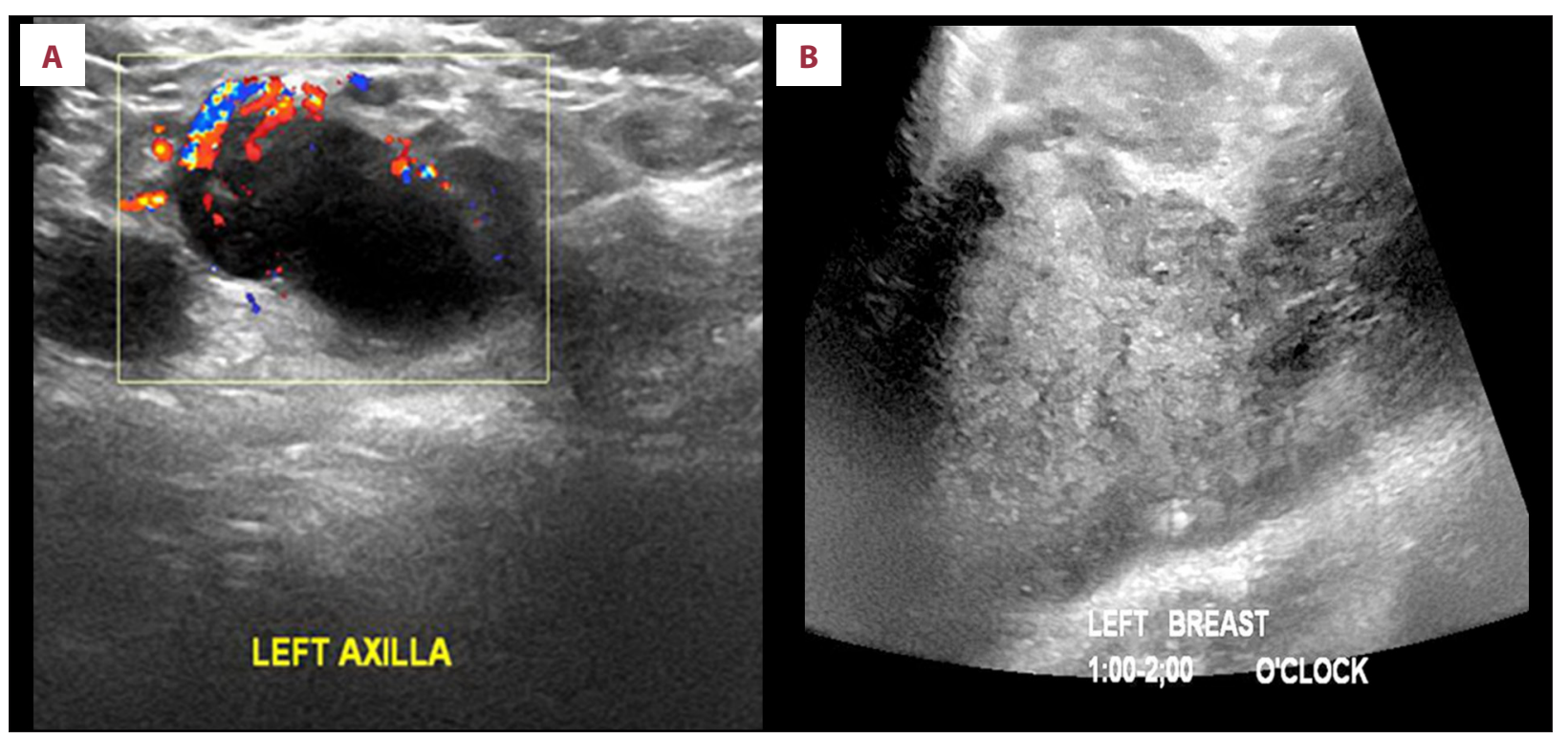

Figure 1. (A, B) Ultrasonography revealed a large ill-defined dense mass lesion with internal increased vascularity in color Doppler imaging. 


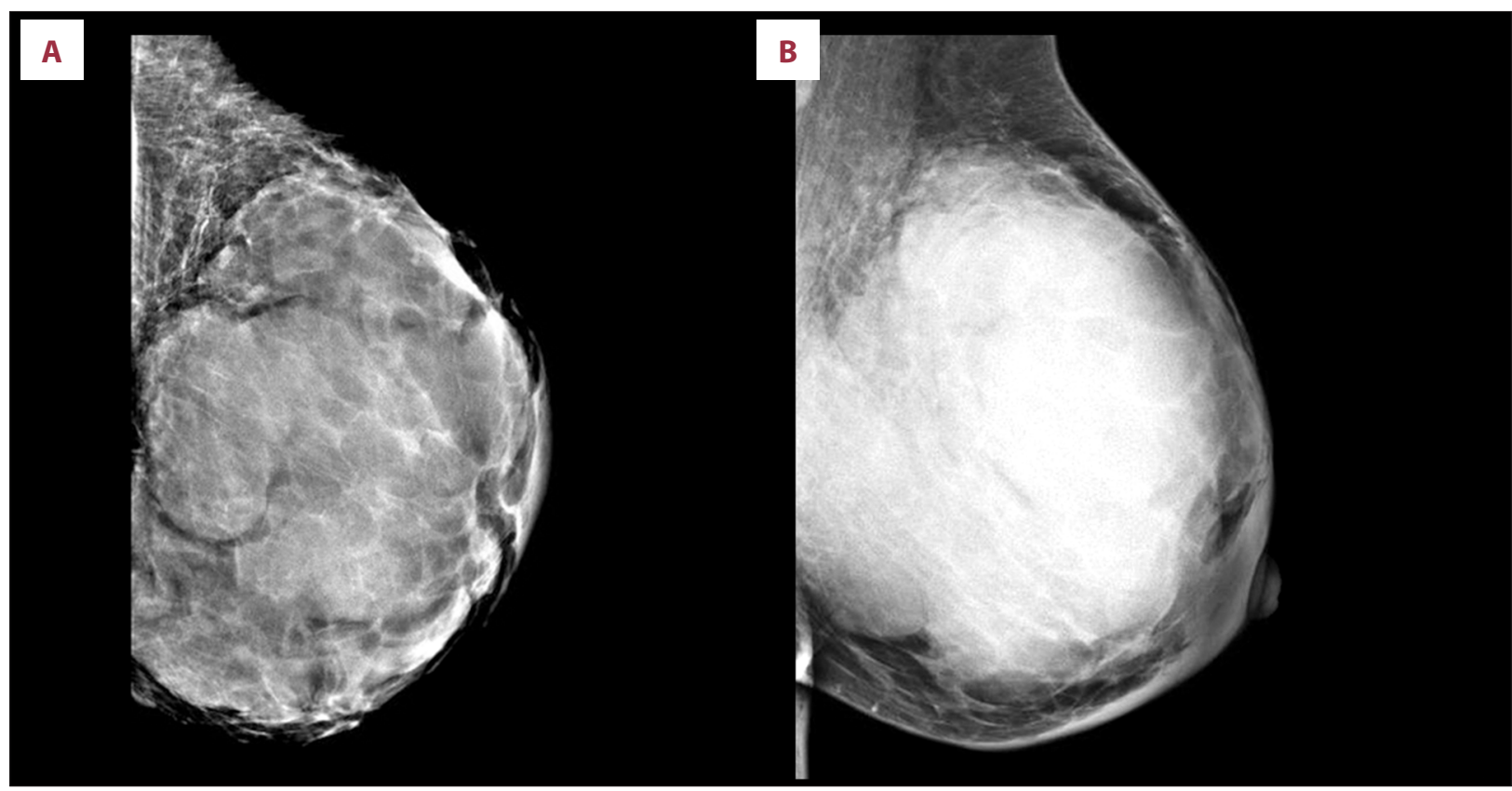

Figure 2. Mammography findings are suggestive of breast cancer with metastatic axillary lymph nodes.

and MUM-1. Staining for CD10 was weak and uncertain. The neoplastic cells were negative for CD3 and CD5, which highlight scattered small T-cells in the background. The proliferation index was approximately $70 \%$.

In summary, the patient had diffuse large B-cell lymphoma, stage IV B, with bulky disease (multiple enlarged lymph nodes at left axillary, left subclavicular, splenic hilum, retroperitoneal, and mesenteric regions) and multiple extranodal localization (breast, muscle, and lung).

The case was discussed in the breast MDT meeting which recommended the treatment with 4 cycles of combination chemotherapy with R-CODOX/IVAC.

R-CODOX-M regimen included rituximab, cyclophosphamide, vincristine, and doxorubicin. The R-IVAC regimen consisted of rituximab, etoposide, ifosfamide, and cytarabine. After chemotherapy, there was a persistent left breast mass, but a PET/CT scan showed no significant uptake. Breast biopsy showed myxomatous and fibrous tissue. The patient was then scheduled for radiotherapy (20 fractions). Two months post-treatment, $\mathrm{PET} / \mathrm{CT}$ showed a favorable response, resolution of PDG avid lymphadenopathy, and around $90 \%$ decrease in PDG avidity of the breast. Intrathecal cytarabine was given as a CNS prophylaxis. By completing the recommended chemo- and radio-therapy, the patient remained in a remission status. The patient had 3 PET scans: at presentation, after the 2 nd therapy course, and 3 weeks after the $4^{\text {th }}$ course. Figure 6 shows a follow-up PET scan that revealed a radiologically favorable response in terms of non-significant mild
F-18 fluorodeoxyglucose (FDG) uptake at the periphery of the residual left breast mass.

\section{Discussion}

Primary breast lymphoma (PBL) remains an infrequent neoplasm, so clinicians treating breast carcinomas should be aware of this entity in order to distinguish its clinical presentation, management, and prognosis from breast carcinoma cases [10]. With the development of newer diagnostic modalities, the incidence of PBL is increasing, which substantiates the importance of clinical information and awareness of this rare disease. It is predominantly (95\%-100\%) reported among female patients and is very rare in males [8]. Single-breast involvement is more common, particularly in the upper quadrant of the right side $[9,11]$. Approximately $1 \%$ to $14 \%$ of all PBL cases have bilateral breast lymphomas [12]. In our patient, the breast lump was detected on the left side. PBL can be classified based on the presenting symptoms as type $A$ (atypical symptoms) and type B (fever, night sweats, $10 \%$ weight loss within half a year) [8]. The investigational findings of our case showed large B-cell lymphoma (non-germinal center type). However, the lower incidence and atypical clinical manifestations makes it difficult to discriminate from breast carcinoma without confirmation by pathological biopsy and immunohistochemical staining $[8,13]$. Of note, the rapid increase in the breast mass size should be first considered as PBL.

Based on the histopathological findings, breast tumors can be categorized as large cell B-cell lymphomas, monocytoid B-cell 


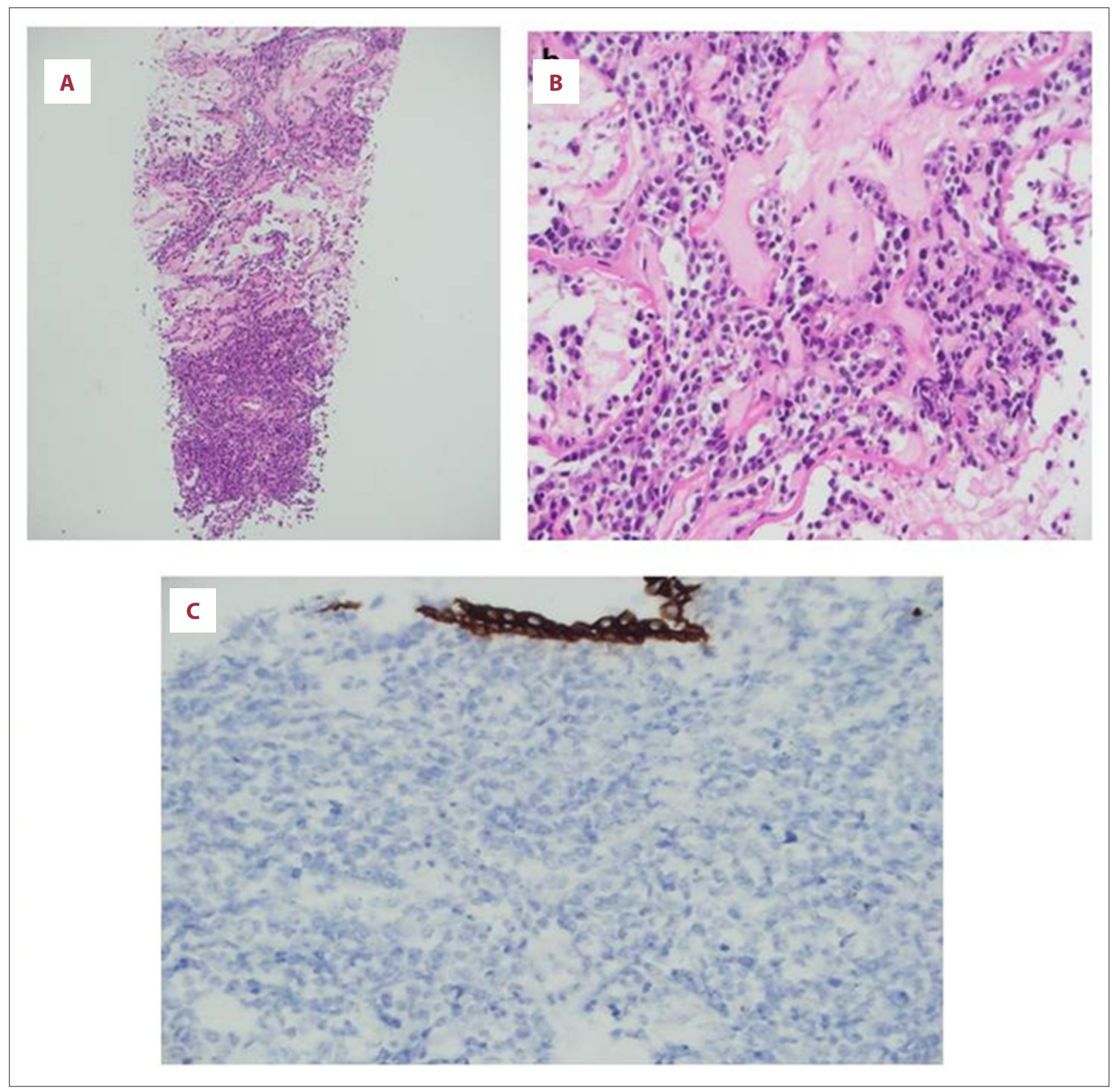

Figure 3. Core biopsy (A) low power magnification (100 H\&E stain) showed diffuse cellular infiltrate. (B) High power magnification (400 H\&E stain) showed large lymphocyte cells with prominent nuclei. (C) Pankeratin AE1/AE3 (epithelial marker) showed normal duct in the section (central plastic) diffuse large cell.

lymphomas (MBCL), and undifferentiated [14]. It has been reported that the majority of PBL cases have a B-cell lineage and diffuse large B-cell lymphoma (DLBCL), which accounts for $40-70 \%$ of all breast lymphomas with features of non-germinal center cells [8]. The assessment of our case revealed DLBCL of the breast with multiple extranodal presentations, which is consistent with the current literature. The diagnostic approach of PBL cases includes radiological investigation (mammography, ultrasonography, magnetic resonance imaging, and positron emission tomography), fine-needle aspiration cytology (FNAC), and immunohistochemical biomarkers [15]. In the present case, FNA of the left axillary lymph node showed atypical lymphoid cells suggestive of malignancy. The immunohistochemistry profile was consistent with diffuse large B-cell lymphoma, non-germinal center-type. The malignant lymphoid cells were positive for the B-cell markers CD20 and CD79 and negative for T-cell markers CD3 and CD5. FNAC is ideal for the primary diagnosis of $\mathrm{PBL}$ as it helps in early detection and treatment. However, the differential diagnosis of PBL should be made according to the pathological findings [16], which is crucial for understanding the typing and staging of these tumors. To distinguish PBL based on its origin from the immune tissues, 


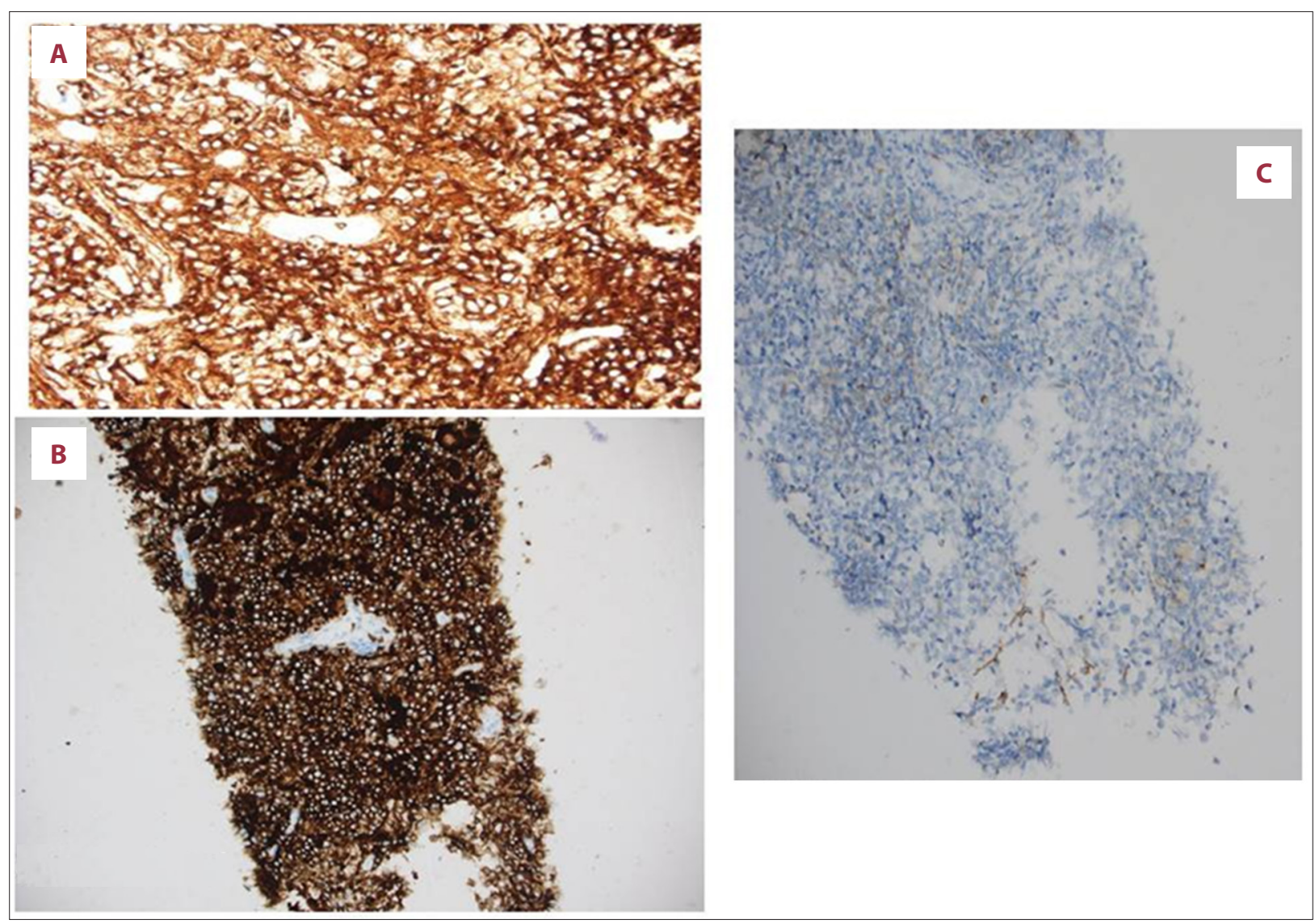

Figure 4. Immunohistochemistry. (A) CD20 (positive); (B) CD79a (posititive); (C) CD10 (weak/negative).

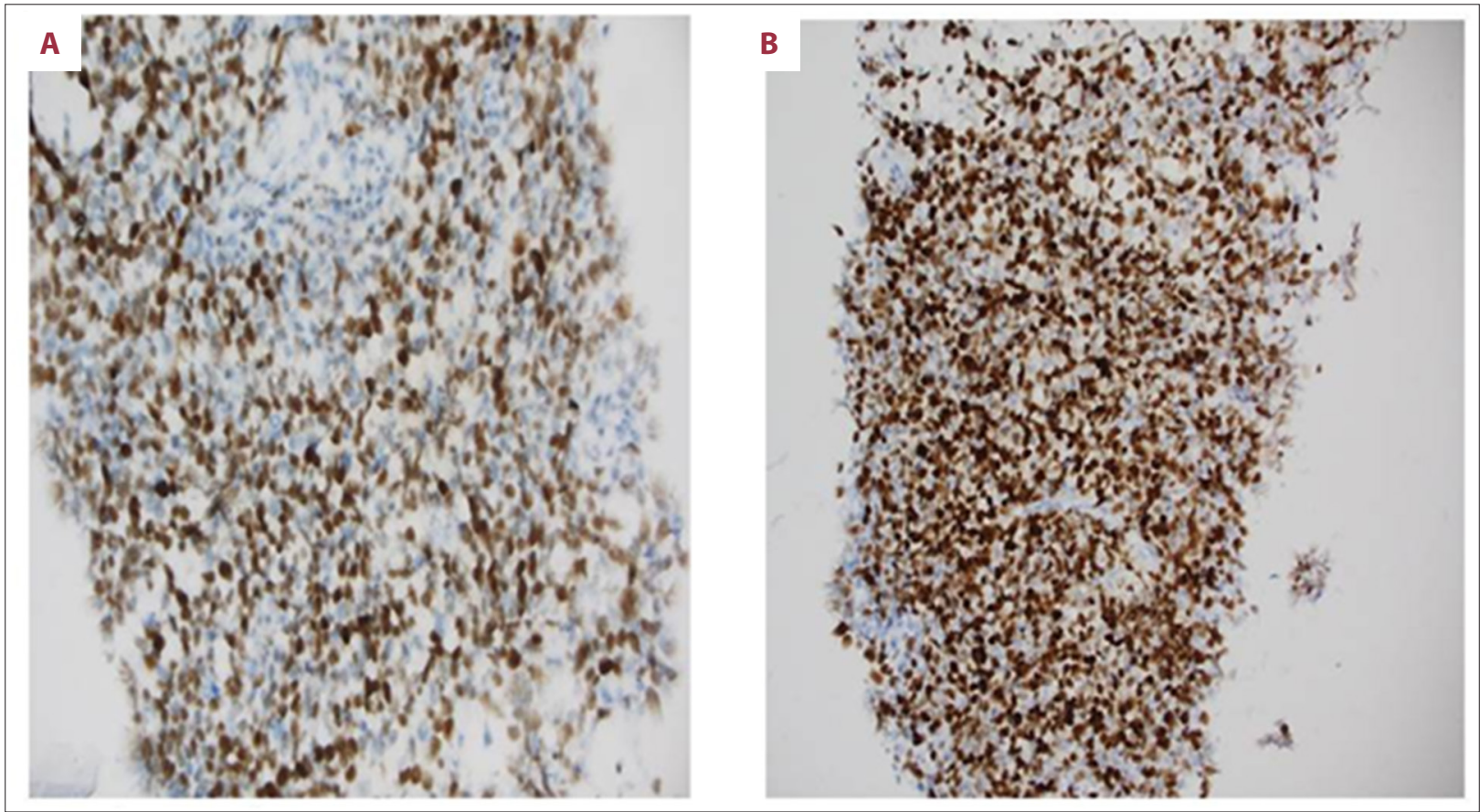

Figure 5. Immunohistochemistry. (A) BCL6 (positive); (B) MUM-1 (posititive). 


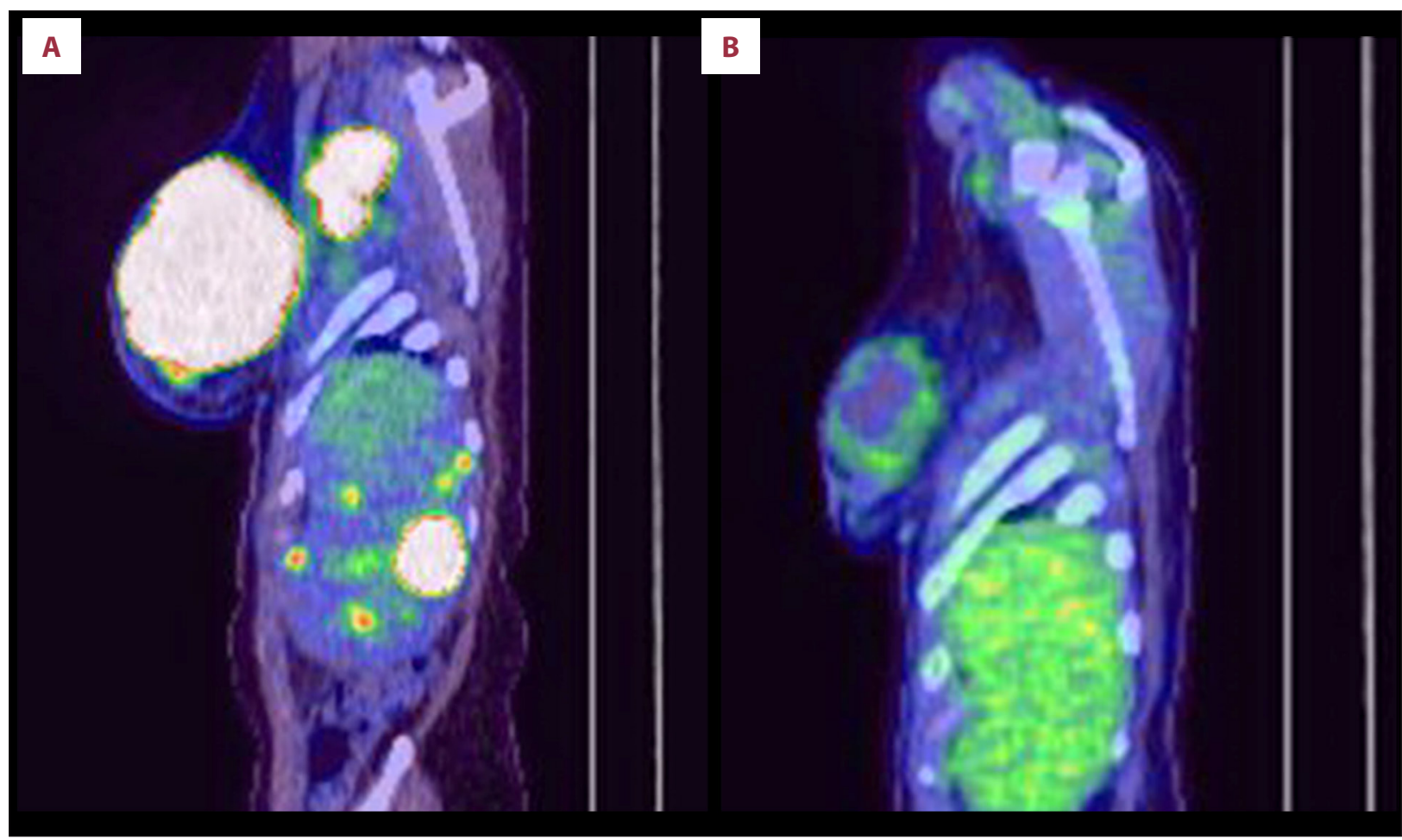

Figure 6. PET scan (A) initial scan showed intense hypermetabolism in the left breast DLBCL (B) follow-up PET scan revealed nonsignificant mild FDG uptake at the periphery of the residual left breast mass after treatment.

these tumors can be accurately classified as originating from the hematopoietic lymphatic or epithelial systems. Moreover, lymphoma evaluation and management are dramatically improving due to the rapidly growing database on gene expression patterns and protein expression [17]. Recently, Ondrejka and Hsi summarized the essential data on pathogenesis, clonal or tumoral evolution, and identification of the biomarkers that may be useful for prognostic or therapeutic strategy in lymphomas patients [18].

The management strategies for PBL vary broadly, from surgical intervention to combination chemotherapy and radiotherapy [19]. However, there is no up to date standard guideline for PBL treatment. Mastectomy for PBL is not well-supported be-cause it shows neither improved survival nor reduced risk of re-currence [20-22]. Several studies have recommend-ed that surgery should be offered for diagnosis only and that minimally invasive surgery is the preferable option because extensive surgery may carry a high risk of morbidity. Also, axillary dissection adds no therapeutic advantage [20-22].

At present, early stages of PBL can be successfully treated with combination therapy, in which $\mathrm{CHOP}$ regimen is the most common chemotherapeutic agent [23]. Several investigators have recommended a treatment regimen involving combination chemotherapy with or without radiation therapy $[6,19]$. Consistent with these studies, our patient was treated with 4 cycles of combination chemotherapy with R-CODOX/IVAC along with radiotherapy [24-26]. The patient presentation was aggressive with a stage IV BE (PET scan at presentation showed lesions in the lung, spleen, and multiple lesions in the bone). The size of the mass was $7 \mathrm{~cm}$ and lactate dehydrogenase level was high. The age-adjusted international prognostic index (aalPI) was high (score=3). Therefore, our patient was at high risk because 3 prognostic factors were present (LDH, performance, and staging). It is well known that CNS relapse is common in this type of lymphoma. Therefore, our MDT decided to start with an aggressive chemotherapy regimen (R-CODOX-M/RIVAC) containing high-dose methotrexate, which can penetrate the blood-brain barrier, followed by involved-field radiotherapy (IFRx). There is currently no consensus on the treatment of this subtype of lymphoma; however, combining radiotherapy with chemotherapy is considered the most successful therapy. The risk of CNS involvement and relapse are high; most authors believe that addition of CNS-directed treatment is essential $[27,28]$. Many authors believe that this subtype of disease is associated with poor prognosis and is considered an unfavorable primary site.

This is a novel case report from our region in the Middle East, as no studies have been published yet regarding breast lymphoma and its frequency in this part of the world. With the recent implementation of the breast MDT and discussion of breast cancer cases in a weekly MDT involving all the relevant 
specialties, we have started to investigate many aspects of breast cancer in Qatar. Therefore, this case report paves the way to explore breast lymphoma management, outcomes, and prognosis.

\section{Conclusions}

This is an unusual case of non-germinal (activated B-cell type) breast lymphoma in a woman with a painless mass in the left breast. Screening and diagnosis of PBL based on advanced radiological investigations are useful for identification of this unusual neoplasm. Moreover, early and accurate diagnosis of PBL is crucial for selecting the appropriate treatment strategies to avert potentially harmful surgical interventions. This case

\section{References:}

1. Lokesh KN, Sathyanarayanan V, Lakshmaiah KC et al: Primary breast diffuse large B cell lymphoma - report of 6 cases from South India with review of literature. Indian J Surg Oncol, 2013;4(4): 368-73

2. Wiseman C, Liao KT: Primary lymphoma of the breast. Cancer, 1972; 29(6): 1705-12

3. Hugh JC, Jackson FI, Hanson J, Poppema S: Primary breast lymphoma. An immunohistologic study of 20 new cases. Cancer, 1990; 66: 2602-11

4. Surov A, Holzhausen $\mathrm{HJ}$, Wienke A et al: Primary and secondary breast lymphoma: prevalence, clinical signs and radiological features. Br J Radiol, 2012; 85(1014): e195-e205

5. Sabaté JM, Gómez A, Torrubia S et al: Lymphoma of the breast: clinical and radiologic features with pathologic correlation in 28 patients. Breast J, 2002; 8(5): 294-304

6. Cox J, Lunt L, McLean L: Haematological cancers in the breast and axilla: a drop in an ocean of breast malignancy. Breast, 2005; 14(1): 51-56

7. Domchek SM, Hecht JL, Fleming MD et al: Lymphomas of the breast: primary and secondary involvement. Cancer, 2002, 94: 6-13

8. Yang H, Lang R-G, Fu L: Primary breast lymphoma (PBL): A literature review. Clin Oncol Cancer Res, 2011; 8: 128-32

9. Inic Z, Inic M, Zegarac M et al: Three cases of combined therapy in primary breast lymphoma (PBL) with successful outcomes. Clin Med Insights Oncol, 2013; 7: 159-63

10. Zhao YF, Jiao F, Liang HQ et al: Primary malignant non-Hodgkin's lymphoma of the breast: A case report. Oncol Lett, 2014; 8(6): 2597-600

11. Garg NK, Bagul NB, Rubin G, Shah EF: Primary lymphoma of the breast involving both axillae with bilateral breast carcinoma. World I Surg Oncol, 2008; 6: 52

12. Jennings WC, Baker RS, Murray SS et al: Primary breast lymphoma: the role of mastectomy and the importance of lymph node status. Ann Surg, 2007; 245: 784-89

13. Domchek SM, Hecht JL, Fleming MD et al: Lymphomas of the breast: primary and secondary involvement. Cancer, 2002; 94: 6-13

14. Hugh JC, Jackson Fl, Hanson J, Poppema S: Primary breast lymphoma. An immunohistologic study of 20 new cases. Cancer, 1990; 66(12): 2602-11

15. Cavalli F, Stein H, Zucca E: Extranodal lymphomas: Pathology and management. Informa Healthcare 2008. Retrieved from http://informahealthcare. com/doi/book/10.3109/9780203091753 accessed on August 18, 2015 report opens the way for early diagnosis, appropriate treatment and favorable outcomes of breast lymphoma.

\section{Acknowledgment}

The authors thank all the general surgery staff for their cooperation.

\section{Statement}

The authors have no conflict of interests and no financial issues to disclose. This case report has been approved by the Medical Research Center (IRB \#15270/15), Hamad Medical corporation, Doha, Qatar.

16. Schwartz IS, Stranchen JA: Lymphocytic mastopathy: An antoimmune disease of the Breast? Am J Clin Pathol, 1990; 93: 725-30

17. Armitage J: Staging Non-Hodgkin Lymphoma. Cancer J Clin, 2005; 55: 368-76

18. Ondrejka SL, Hsi ED: Pathology of B-cell lymphomas: diagnosis and biomarker discovery. Cancer Treat Res, 2015; 165: 27-50

19. Nagata S, Nishimura A, Iwashita $Y$ et al: Primary breast lymphoma in the right breast during treatment for left breast cancer. World J Surg Oncol, 2007; 5: 134

20. Jennings WC, Baker RS, Murray SS et al: Primary breast lymphoma: the role of mastectomy and the importance of lymph node status. Ann Surg, 2007. 245: 784-89

21. Ryan G, Martinelli G, Kuper-Hommel $M$ et al: Primary diffuse large B-cell lymphoma of the breast: prognostic factors and outcomes of a study by the International Extranodal Lymphoma Study Group. Ann Oncol, 2008; 19: 233-41

22. Uesato M, Miyazawa Y, Gunji Y et al: Primary non-Hodgkin lymphoma of the breast: report of a case with special reference to 380 cases in the Japanese literature. Breast Cancer, 2005; 12: 154-58

23. Mouna B, Saber B, Tijani EH et al: Primary malignant non-Hodgkin's lymphoma of the breast: a study of seven cases and literature review. World J Surg Oncol, 2012; 10: 151

24. Phan J, Mazloom A, Jeffrey Medeiros L et al: Benefit of consolidative radiation therapy in patients with diffuse large B-cell lymphoma treated with R-CHOP chemotherapy. J Clin Oncol, 2010; 28(27): 4170-76

25. Doolittle ND, Abrey LE, Shenkier TN et al: Brain parenchyma involvement as isolated central nervous system relapse of systemic non-Hodgkin lymphoma: an International Primary CNS Lymphoma Collaborative Group report. Blood, 2008; 111(3): 1085-93

26. Mohamedbhai SG, Sibson K, Marafioti T et al: Rituximab in combination with CODOX-M/IVAC: a retrospective analysis of 23 cases of non-HIV related B-cell non-Hodgkin lymphoma with proliferation index $>95 \%$. $\mathrm{Br}$ Haematol, 2011; 152(2): 175-81

27. Arkenau HT, Chong G, Cunningham D et al: The role of intrathecal chemotherapy prophylaxis in patients with diffuse large B-cell lymphoma. Ann Oncol, 2007; 18(3): 541-45

28. Boehme V, Zeynalova S, Kloess $M$ et al: Incidence and risk factors of central nervous system recurrence in aggressive lymphoma - a survey of 1693 patients treated in protocols of the German High-Grade Non-Hodgkin's Lymphoma Study Group (DSHNHL). Ann Oncol, 2007; 18(1): 149-57 